\title{
Autophagy and multivesicular body formation in blastocysts during the experimental diapause in mice
}

\author{
HJ Lim ${ }^{1,2}$, H Shin², J Hyun Jun ${ }^{3}$ and H Song ${ }^{4}$ \\ ${ }^{1}$ Department of Veterinary Medicine, Konkuk University, Seoul, Korea \\ ${ }^{2}$ Department of Biomedical Science \& Technology, Institute of Biomedical Science and \\ Technology, Konkuk University, Seoul, Korea \\ ${ }^{3}$ Department of Biomedical Laboratory Science, Eulji University, Seongnam, Gyeonggi-do, \\ Korea; Department of Senior Healthcare BK21 plus program, Graduated School, Eulji \\ University, Daejoen, Korea \\ ${ }^{4}$ Department of Biomedical Science, CHA University, Seongnam, Gyeonggi-do, Korea
}

Corresponding author e-mail: $\underline{\text { hlim@konkuk.ac.kr }}$

\begin{abstract}
In experimentally induced diapause model in mice, blastocysts remain dormant for an extended period but resume implantation competency upon estrogen injection. The underlying mechanism by which extended longevity of dormant blastocysts is maintained is unclear. We have previously shown that dormant blastocysts, during experimentally induced diapause, exhibit heightened autophagic activation. Activation of autophagy appears to be a crucial adaptive response for survival in the unfavorable uterine environment, as inhibiting autophagy reduces the survival rate of dormant blastocysts. As a unique cell biological change occurring following estrogen supplementation to activate dormant blastocysts, multivesicular bodies (MVBs) accumulate in the trophectoderm. In various cellular contexts, autophagy and MVB formation are linked cell biological phenomena. Herein, we discuss the implications of these cell biological changes in dormant and activated blastocysts.
\end{abstract}

\section{Introduction}

The hormonal requirement for blastocyst implantation is thought to differ among various species [1]. In rodents, estrogen is needed to prepare the uterus to become receptive and activate blastocysts for the two-way interaction between the uterine epithelium and trophectoderm [2]. In this case, estrogen oversees molecular and cellular changes in the uterus and blastocyst by up-or downregulating certain molecules and pathways [3,4]. The obvious need for estrogen in implantation in mice makes it possible to manipulate implantation timing. Ovariectomy (OVX) performed prior to the secretion of preimplantation estrogen on day 4 of pregnancy produces an artificial diapause model in which the timing of blastocyst activation is regulated by exogenous estrogen supplementation [5]. The postpartum mouse with an immediate second pregnancy also enters facultative diapause during lactation, which persists for several days to weeks depending on the number of suckling pups [6].

The experimental diapause model in mice has widely been used to delineate the molecular and cellular mechanisms of the sustainable nature of blastocyst survival during diapause [7]. Although developmental and physiological conditions of this diapause model differ dramatically from species with seasonal diapause [6], the common question hovers around the mechanism as to 
how dormant blastocysts maintain developmental integrity for a long time. The OVX model of dormancy in mice is easily manipulatable and provides leverage to devise fine-controlled experiments to understand the molecular and cellular mechanisms governing the state of dormancy.

In this report, we discuss two cell biological pathways that have been implicated in blastocyst dormancy and activation in mice.

\section{Blastocyst dormancy and autophagy}

Macroautophagy (autophagy thereafter) is a major cellular catabolic pathway that degrades various organelles, macromolecules, and certain pathogens [8]. This process is crucial for the maintenance of cellular homeostasis by recycling organelles and macromolecular building blocks. Building blocks exiting the autophagic process enter various biochemical pathways to produce new macromolecules or ATP [8]. This is the primary intracellular mechanism involved in degrading and recycling long-lived proteins and organelles [9]. An assortment of autophagy (Atg) proteins and associated factors are involved in regulating and executing autophagy [9]. While autophagy is typically ongoing at a basal level within cells, a suboptimal condition surrounding the cells or tissues is a cue for increasing the rate of autophagy. Depending on the cell or tissue types, the description of suboptimality is diverse. Deprivation of certain growth factors or hormones, crowding, hypoxia, or disease conditions is indicated $[8,10,11]$. As autophagy is invariably required for cell survival and adaptation, the autophagic rate is constantly regulated by reflecting changes occurring in the intra- and extracellular environments. Autophagy is referred to as a "doubleedged sword" in some contexts, as it is necessary for cell survival but excess levels can cause cell death [12].

The idea that autophagy may be active during the prolonged survival of dormant blastocysts was first ignited by the work done in Caenorhabditis elegans ( $C$. elegans) worm, a widely used model organism in developmental biology. These worms exhibit a status of dormancy when they face limited food, increased temperature, or other unfavorable conditions [13]. Such dauer worms halt their developmental program and exhibit extended longevity, low metabolic rate, and arrested development [14]. Dauer formation in C. elegans is associated with heightened autophagy and requires $C$. elegans orthologs of the yeast autophagy genes [15]. Extended longevity, lowered metabolism, and arrested development are also the characteristics of observed in dormant blastocysts [16]. The obvious similarities between the two entities indicate that autophagy is involved in maintaining the survival in dormant blastocysts. The gene expression profiles of dormant and activated blastocysts showed that components of autophagy are upregulated at dormancy but decrease upon blastocyst activation $[4,17,18]$. Autophagic activation, assessed in GFP-LC3 transgenic mice, a widely-used model for in vivo imaging of autophagy [19], was observed in dormant blastocysts both in the inner cell mass (ICM) and trophectoderm. In these transgenic mice, GFP-LC3 transgene is expressed in all cells. The fusion protein can be incorporated in the autophagic process, and form cytoplasmic green puncta of various sizes. These green dots are considered as initial autophagic structures called autophagosomes [20]. Autophagosomes then fuse with lysosomes for the degradation of enclosed contents [19]. Dormant blastocysts expressing GFP-LC3 transgene, after 3.5 or 9.5 days of dormancy, showed accumulation of GFPLC3 puncta [17]. Whether the length of dormancy increases the extent of autophagic activation cannot be determined, as this is a qualitative assay. Upon blastocyst activation by injection of estrogen, autophagic puncta were still observed in the trophectoderm of activated blastocysts. Interestingly, these puncta cleanly disappeared from the ICM portion within $12 \mathrm{~h}$ of estrogen injection. Considering that the ICM carries the cells of the embryo proper, rapid downregulation of 
autophagy in this cell type may be crucial for minimizing cellular damage that may have occurred during the long period of autophagic activation. Three-methyladenine (3-MA) is an inhibitor of class III phosphoinositide 3-kinase which produces phosphatidylinositol 3-phosphate (PI3P). As PIP3 is required for autophagosome formation and maturation, 3-MA is considered to inhibit early steps in the autophagic process [21]. In vivo injection of 3-methyladenine (3-MA) during diapause reduced the number of surviving dormant blastocysts, supporting the role of autophagy in embryonic survival [17].

The survival rate of dormant blastocysts is inversely correlated with the length of dormancy in OVX-induced diapause model in mice [22]. Developmental competency after activation and implantation shows a similar association, as both implantation rate and average fetal weight show a decreasing tendency in correlation with the length of dormancy [17]. While a compromise in developmental competency after a long diapause in mice is evident, this model, induced under specific suboptimal conditions, is artificial and does not reflect the situation during physiologic diapause [6]. In mice, the length of lactational diapause observed in most postpartum mice is generally between 1-7 days [23], making it similar to the model of short-term diapause (see Figure 1).

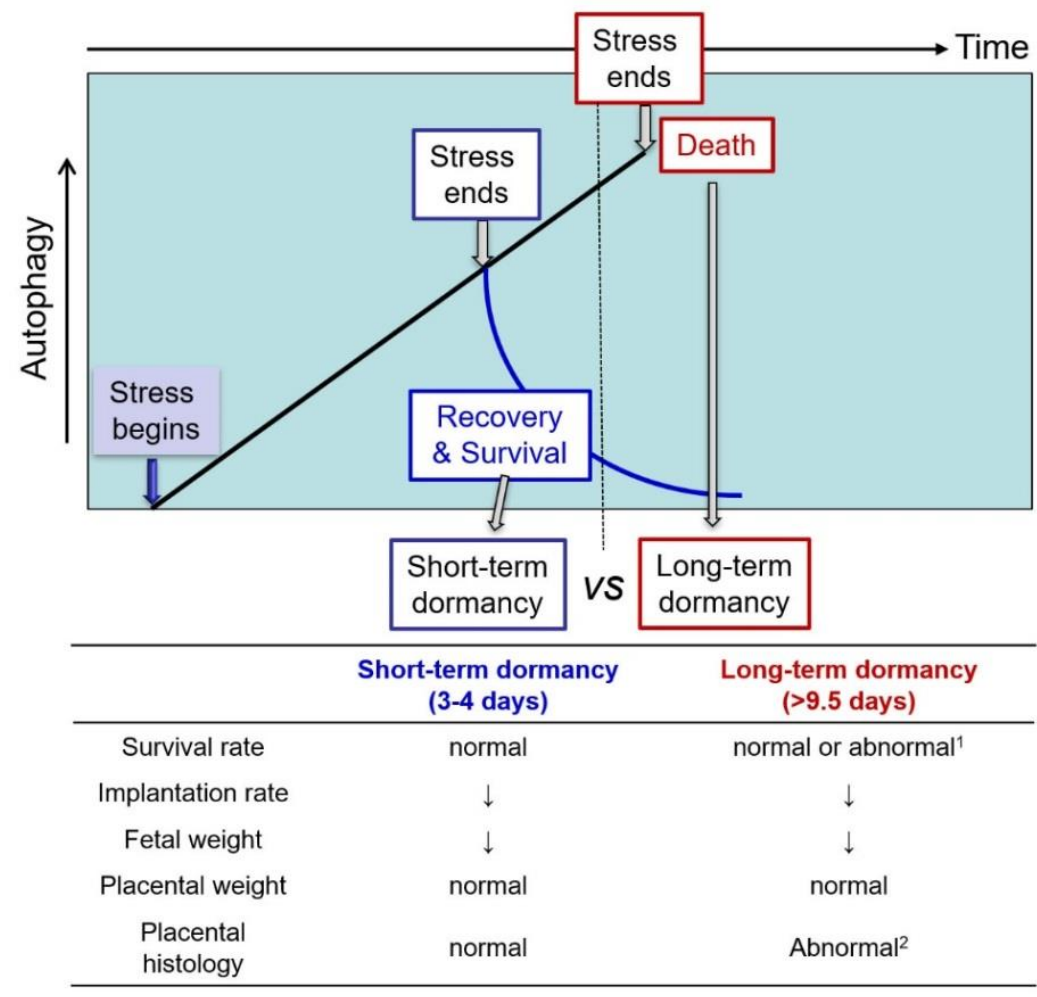

Figure 1. When autophagy affects developmental competency, the duration matters in mice.

Autophagy is ongoing in cells at a basal level but certain cell type-specific suboptimal conditions, depicted as "stress" in the diagram, can increase the autophagic rate. If the stress is short-term, cells may recover and survive with negligible damage. If the stress goes on for an extended period, cellular damage may become too extensive to repair. Under this condition, a compromise between developmental competency or cell death may occur. In the experimental diapause model in mice, the stress would be the lack of preimplantation estrogen. Short-term dormancy of 3-4 days does not appear to influence survival and development of activated blastocysts, whereas long-term dormancy of 10 days or longer leads to the manifestation of intrauterine growth retardation accompanied by placental defects [17]. The length of lactational diapause in mice was 1-7 days in $90 \%$ of postpartum mice [23], and thus closer to short-term diapause depicted herein. 
${ }^{1}$ In more than 30 days of delayed implantation, the survival rate of dormant blastocysts was dramatically reduced [22]. When an autophagy inhibitor is administered, the mouse survival rates decrease even in short-term diapause. ${ }^{2}$ The abnormalities in placental histology were noted in poorly developed fetal vessels in the labyrinth and chorionic plate of fetal origin [17].

\section{Blastocyst activation and multivesicular body formation}

In the experimental diapause model in mice, implantation resumes when a small amount of estrogen is injected to delayed implanting mice [2]. The requirement for estrogen in blastocyst activation is absolute and highly efficient; it was shown that $3 \mathrm{ng}$ of $17 \beta$-estradiol $\left(E_{2}\right)$ injection is sufficient to induce implantation [24]. For blastocyst activation, the conversion of $E_{2}$ to 4-hydroxy$E_{2}$ by the cytochrome $\mathrm{P} 4501 \mathrm{~B} 1$ enzyme is required, as no implantation occurs when the activity of this enzyme was inhibited [25].

MVBs are the intermediate endosomal structures characterized by the presence of multiple intraluminal vesicles [26]. In the biogenesis of MVBs with internalized vesicles, the sequential actions by the endosomal sorting complex required for transport (ESCRT) complexes are required [27]. During MVB biogenesis, ubiquitinated cargoes are sorted to intraluminal vesicles for lysosomal degradation by a process involving ESCRT factors [28]. Fusion between the MVB and autophagosome produces a structure called amphisome, which either fuses with a lysosome or the plasma membrane [29]. Fusion of an amphisome and lysosome invariably leads to degradation of autophagic debris [30].

In activated blastocysts of mice and rats, these peculiar subcellular structures, MVBs, appear in the trophectoderm (see Figure 2A). Their presence was confirmed in several ultrastructural analyses of activated blastocysts in OVX-induced diapause models of rodents [17 31-33], but not reported in the activated blastocysts of skunks [34] or roe deer [35] with seasonal diapause. MVBs are also not readily visible in normal implanting blastocysts [17]. Our unpublished findings (see Figure 2 B\&C) suggest that blastocysts during the lactational diapause in mice (day 8 of postpartum pregnancy) showed some MVB accumulation. Leukemia inhibitory factor (LIF) is a uterine protein required for implantation, and Lif $^{/-}$female mice show complete failure of implantation [36]. In the uteri of pregnant Lif $^{-/}$mice after the time of implantation, blastocysts remain in a free-floating state. These blastocysts become larger and more elongated over time, indicating that they have certain features of activated blastocysts. These blastocysts also show numerous MVBs in the trophectoderm (see Figure $2 \mathrm{D \& E}$ ). Whether the status of MVB formation is a usable index of blastocyst activation requires further studies.

Fusion of MVBs with the plasma membrane leads to the release of intraluminal vesicles as exosomes. Exosome are recognized as a mechanism of intercellular communication [37]. Additionally, inhibition of MVB formation was shown to induce autophagosome accumulation within cells, gradually leading to cell death [38]. Thus, MVB formation is required for removing autophagic debris. While MVBs are versatile structures, cells with heightened MVB accumulation share common features; they are cleaning up. For example, numerous MVBs are detected during mass cleaning processes in the cytoplasm of reticulocytes, in which most cytoplasm is discarded to produce mature red blood cells [39]. Additionally, the increased autophagic activation demands MVBs during the removal of accumulated autophagic vacuoles $[29,38]$. 

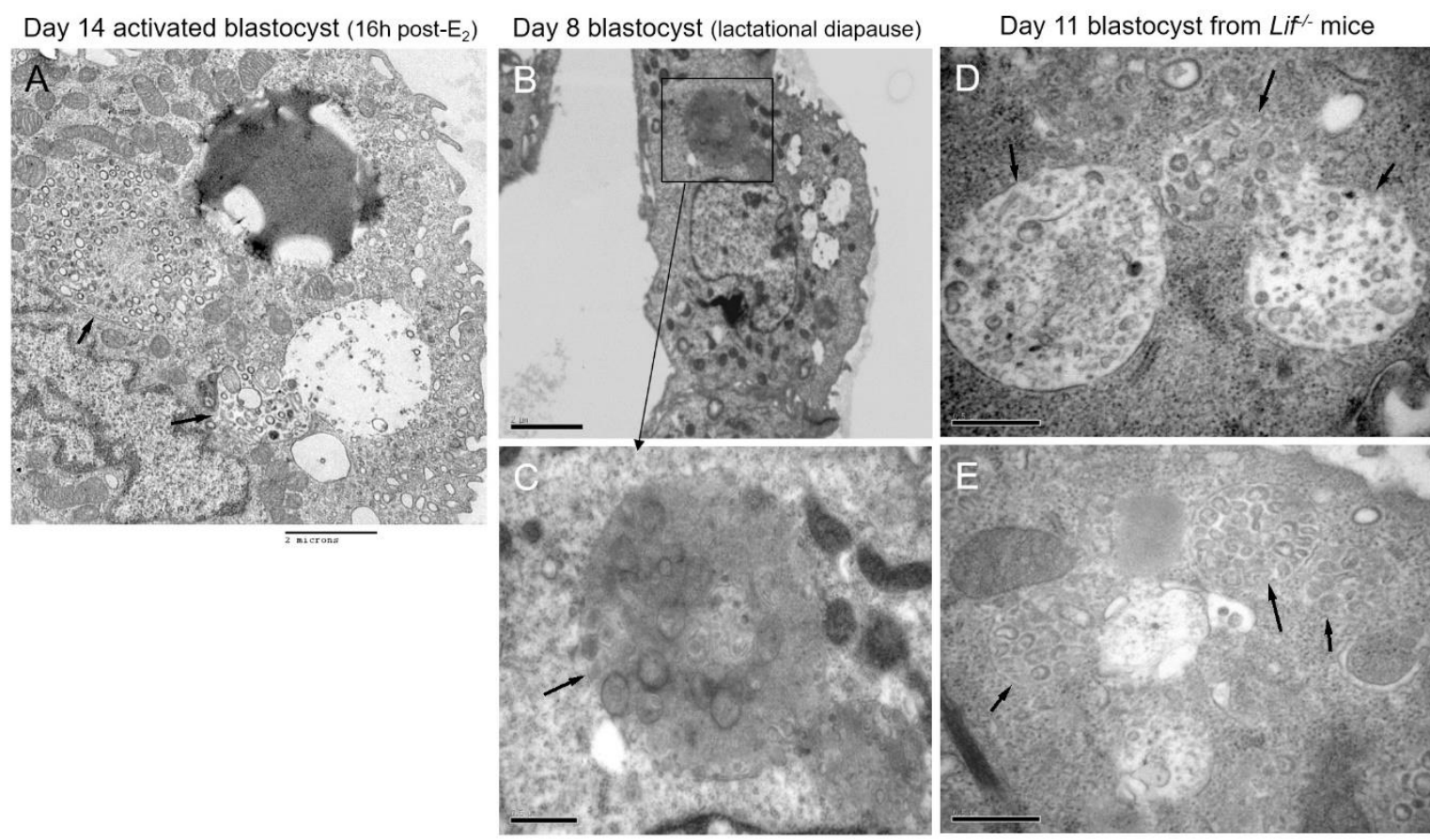

Figure 2. Ultrastructural analyses of various mouse blastocysts with multivesicular body formation. Unpublished data.

A. Region of a trophoblast in day 14 activated blastocyst $16 \mathrm{~h}$ post- $E_{2}$. MVBs are indicated with arrows. Scale bar $=2 \mu \mathrm{m}$.

B. Region of a trophoblast in day 8 blastocyst during lactational diapause. A black rectangle area is enlarged in C. Scale bar $=2 \mu \mathrm{m}$.

C. Multivesicular body (MVB) is shown (arrow). Scale bar $=0.5 \mu \mathrm{m}$.

D\&E. Enlarged areas of a blastocyst from day 11 pregnant Lif $^{-/}$female mice. MVBs are indicated with arrows. Scale bar $=0.5 \mu \mathrm{m}$.

Increased autophagy is the characteristic of dormant blastocysts from OVX-induced diapause [17], whereas the increased MVB formation is that of activated blastocysts [40]. Given the strong link between these processes found in other systems, an investigation of the association between autophagy and MVBs in blastocyst activation was conducted [40]. The identity of MVBs was first confirmed using several molecular markers of MVBs. Among the long list of ESCRT components [41], the tumor susceptibility gene 101 (Tsg101), the core component of ESCRT I complex, was used as a marker in our study because of its central role in targeting ubiquitinated cargoes to MVBs [28]. Lysobisphosphatidic acid (LBPA) is a phospholipid specifically found in the intraluminal vesicles within MVBs [42], thus considered a specific MVB marker. 1,1'-Dioctadecyl-3,3,3', $3^{\prime}$ tetramethylindocarbocyanine iodide (Di-I), is a dye for live imaging of MVBs. Using these markers, we found that activated blastocysts, but not dormant or normal implanting blastocysts (day 4.5 of pregnancy), accumulate MVBs [40]. The expression of LBPA was more intense in activated blastocysts with longer dormancy period ( $\sim 9.5$ days) than shorter dormancy period ( 3.5 days). Interestingly, levels of ubiquitin accumulation exhibit a similar tendency, i.e., activated blastocysts with longer dormancy period showed intense ubiquitin accumulation whereas those with shorter dormancy showed little. Whether a prolonged period of autophagic activation renders activated blastocysts with more MVB accumulation was next examined. An inhibitor for autophagy (3-MA) was administered to delayed implanting mice during diapause. Activated blastocysts from 3-MAinjected mice showed lower LBPA signal but increased ubiquitin accumulation, suggesting that 
MVB formation is reduced and ubiquitinated waste accumulate more in activated blastocysts under the inhibition of autophagy [40].

In postimplantation embryo, fibroblast growth factor (FGF) signaling was shown to induce vesicular trafficking in the embryonic node [43]. We tested the possibility if FGF signaling is involved in MVB formation in acviated blastocysts by using PD173074, an FGFR antagonist. PD173074 injection to the uteri during activation of implantation led to a reduction in MVB formation in activated blastocysts, suggesting involvement of FGFR signaling in this process [40].

\section{Conclusion: cell biological changes conveying molecular changes}

We herein discussed two unique cell biological changes observed in mouse blastocysts during OVX-induced diapause and activation. Both autophagic activation and MVB accumulation are not readily observed during normal implantation but heavily presented in this model. Whether similar changes occur during normal implantation with less visibility or in obligatory diapause of other species is currently unknown. MVB-positive signals in activated blastocysts are mostly enriched in the mural trophectoderm, where the implanting blastocyst makes the first contact with the uterine epithelium [40]. While MVBs may be an efficient mechanism for clearing subcellular debris accumulated during prolonged autophagy, exosomal release may also occur to convey specific signals for upcoming implantation. Multiple reports suggest that exosomes are involved in embryo-uterine communications $[44,45]$. Further studies are needed to determine if specific signaling factors are actively targeted to exosomes for secretion. How our findings can be translated in the context of other diapausing animals depends on the species-specific developmental program and physiological setting.

\section{Declaration of interest}

The authors declare that there is no conflict of interest that could be perceived as prejudicing the impartiality of this article.

\section{Funding}

This work was supported by the National Research Foundation of Korea (NRF) grant (NRF2017R1A2B4002932) funded by the Korea government (MSIT).

\section{Acknowledgements}

Authors would like to thank Dr. S. K. Dey for his encouragement to enter the fascinating area of diapause research.

\section{References}

[1] Dey SK, Lim H, Das SK, Reese J, Paria BC, Daikoku T \& Wang H. 2004 Molecular cues to implantation. Endocrine Reviews 25 341-373.

[2] Paria BC, Huet H \& Dey SK. 1993 Blastocyst's state of activity determines the "window" of implantation in the receptive mouse uterus. Proceedings of the National Academy of Sciences U S A 90 10159-10162.

[3] Reese J, Das SK, Paria BC, Lim H, Song H, Matsumoto H, Knudtson KL, DuBois RN \& Dey SK. 2001 Global gene expression analysis to identify molecular markers of uterine receptivity and embryo implantation. Journal of Biological Chemistry 276 44137-44145.

[4] Hamatani T1, Daikoku T, Wang H, Matsumoto H, Carter MG, Ko MS \& Dey SK. 2004 Global gene 

162

expression analysis identifies molecular pathways distinguishing blastocyst dormancy and activation. Proceedings of the National Academy of Sciences U S A 101 10326-10331.

[5] Yoshinaga K \& Adams CE. 1966 Delayed implantation in the spayed, progesterone treated adult mouse. Journal of Reproduction and Fertility 12 593-595.

[6] Renfree MB \& Fenelon JC. 2017 The enigma of embryonic diapause. Development 144 3199-3210.

[7] Wang H \& Dey SK. 2006 Roadmap to embryo implantation: clues from mouse models. Nature Reviews Genetics 7 185-199.

[8] Boya P, Reggiori F \& Codogno P. 2013 Emerging regulation and functions of autophagy. Nature Cell Biology 15 713-720.

[9] Mizushima N. 2018 A brief history of autophagy from cell biology to physiology and disease. Nature Cell Biology 20 521-527.

[10] Klionsky DJ, Abdelmohsen K, Abe A et al. 2016 Guidelines for the use and interpretation of assays for monitoring autophagy (3rd edition). Autophagy 12 1-222.

[11] Kuma A, Komatsu M \& Mizushima N. 2017 Autophagy-monitoring and autophagy-deficient mice. Autophagy 13 1619-1628.

[12] Lim HJ \& Song H. 2014 Evolving tales of autophagy in early reproductive events. International Journal of Developmental Biology 58 183-187.

[13] Golden JW \& Riddle DL. 1984 The Caenorhabditis elegans dauer larva: developmental effects of pheromone, food, and temperature. Developmental Biology 102 368-378.

[14] Hu PJ. 2007 Dauer. WormBook 1-19

[15] Melendez A, Talloczy Z, Seaman M, Eskelinen EL, Hall DH \& Levine B. 2003 Autophagy genes are essential for dauer development and life-span extension in C. elegans. Science 301 1387-1391.

[16] Weitlauf HM. 1974 Metabolic changes in the blastocysts of mice and rats during delayed implantation. Journal of Reproduction and Fertility 39 213-224.

[17] Lee J, Oh H, Song H, Jun JH, Roh JR, Xie H, Dey SK \& Lim HJ. 2011 Autophagy Regulates Embryonic Survival During Delayed Implantation. Endocrinology 152 2067-2075.

[18] He B, Zhang H, Wang J, Liu M, Sun Y, Guo C, Lu J, Wang H \& Kong S. 2019 Blastocyst activation engenders transcriptome reprogram affecting $X$-chromosome reactivation and inflammatory trigger of implantation. Proceedings of the National Academy of Sciences U S A 116 16621-16630.

[19] Mizushima N, Yamamoto A, Matsui M, Yoshimori T \& Ohsumi Y. 2004 In vivo analysis of autophagy in response to nutrient starvation using transgenic mice expressing a fluorescent autophagosome marker. Molecular Biology of the Cell 15 1101-1111.

[20] Mizushima N. 2007 Autophagy: process and function. Genes and Development 21 2861-2873.

[21] Dall'Armi C, Devereaux KA \& Di Paolo G. 2013 The role of lipids in the control of autophagy. Current Biology 23 R33-R45.

[22] Weitlauf HM \& Greenwald GS. 1968 Survival of blastocysts in the uteri of ovariectomized mice. Journal of Reproduction and Fertility 17 515-520.

[23] Mantalenakis SJ \& Ketchel MM. 1966 Frequency and extent of delayed implantation in lactating rats and mice. Journal of Reproduction and Fertility 12 391-394.

[24] Ma WG, Song H, Das SK, Paria BC \& Dey SK. 2003 Estrogen is a critical determinant that specifies the duration of the window of uterine receptivity for implantation. Proceedings of the National Academy of Sciences U S A 100 2963-2968.

[25] Paria BC, Lim H, Wang XN, Liehr J, Das SK \& Dey SK. 1998 Coordination of differential effects of primary estrogen and catecholestrogen on two distinct targets mediates embryo implantation in the mouse. Endocrinology 139 5235-5246.

[26] Wendler F, Bota-Rabassedas N \& Franch-Marro X. 2013 Cancer becomes wasteful: emerging roles of exosomes $(+)$ in cell-fate determination. Journal of Extracellular Vesicles 222390

[27] Wollert T \& Hurley JH. 2010 Molecular mechanism of multivesicular body biogenesis by ESCRT complexes. Nature 464 864-869. 
[28] Sundquist WI, Schubert HL, Kelly BN, Hill GC, Holton JM \& Hill CP. 2004 Ubiquitin recognition by the human TSG101 protein. Molecular Cell 13 783-789.

[29] Fader CM \& Colombo MI. 2009 Autophagy and multivesicular bodies: two closely related partners. Cell Death and Differentiation 16 70-78.

[30] Lefebvre C, Legouis R \& Culetto E. 2018 ESCRT and autophagies: Endosomal functions and beyond. Seminars in Cell and Developmental Biology 74 21-28.

[31] Nilsson O. 1974 The morphology of blastocyst implantation. Journal of Reproduction and Fertility 39 187-194.

[32] Wu JT \& Meyer RK. 1974 Ultrastructural changes of rat blastocysts induced by estrogen during delayed implantation. The Anatomical Record 179 253-272.

[33] Fu Z, Wang B, Wang S, Wu W, Wang Q, Chen Y, Kong S, Lu J, Tang Z, Ran H, Tu Z, He B, Zhang S, Chen Q, Jin W, Duan E, Wang H, Wang YL, Li L, Wang F, Gao S \& Wang H. 2014 Integral proteomic analysis of blastocysts reveals key molecular machinery governing embryonic diapause and reactivation for implantation in mice. Biology of Reproduction 9052.

[34] Enders AC, Schlafke S, Hubbard NE \& Mead RA. 1986 Morphological changes in the blastocyst of the western spotted skunk during activation from delayed implantation. Biology of Reproduction 34 423437.

[35] Aitken RJ. 1975 Ultrastructure of the blastocyst and endometrium of the roe deer (Capreolus capreolus) during delayed implantation. Journal of Anatomy 119 369-384.

[36] Stewart CL, Kaspar P, Brunet LJ, Bhatt H, Gadi I, Köntgen \& Abbondanzo SJ. 1992 Blastocyst implantation depends on maternal expression of leukaemia inhibitory factor. Nature 359 76-79.

[37] Machtinger R, Laurent LC \& Baccarelli AA. 2016 Extracellular vesicles: roles in gamete maturation, fertilization and embryo implantation. Human Reproduction Update 22 182-193.

[38] Filimonenko M, Stuffers S, Raiborg C, Yamamoto A, Malerød L, Fisher EM, Isaacs A, Brech A, Stenmark H \& Simonsen A. 2007 Functional multivesicular bodies are required for autophagic clearance of protein aggregates associated with neurodegenerative disease. Journal of Cell Biology 179 485-500.

[39] Fader CM \& Colombo MI. 2006 Multivesicular bodies and autophagy in erythrocyte maturation. Autophagy 2 122-125.

[40] Shin H, Bang S, Kim J, Jun JH, Song H \& Lim HJ. 2017 The formation of multivesicular bodies in activated blastocysts is influenced by autophagy and FGF signaling in mice. Scientific Reports 741986.

[41] Hurley JH. 2015 ESCRTs are everywhere. The EMBO Journal 34 2398-2407.

[42] Kobayashi T, Stang E, Fang KS, de Moerloose P, Parton RG \& Gruenberg J. 1998 A lipid associated with the antiphospholipid syndrome regulates endosome structure and function. Nature 392 193-197.

[43] Tanaka Y, Okada Y \& Hirokawa N. 2005 FGF-induced vesicular release of Sonic hedgehog and retinoic acid in leftward nodal flow is critical for left-right determination. Nature 435 172-177.

[44] Greening DW, Nguyen HP, Elgass K, Simpson RJ \& Salamonsen LA. 2016 Human Endometrial exosomes contain hormone-specific cargo modulating trophoblast adhesive capacity: insights into endometrial-embryo interactions. Biology of Reproduction 9438

[45] Salamonsen LA, Evans J, Nguyen HP \& Edgell TA. The microenvironment of human implantation: determinant of reproductive success. American Journal of Reproductive Immunology 75 218-225.

\section{Audience Discussion}

Jane Fenelon: I was wondering if you have a reason behind why you think an extended dormancy is bad, because part of the reason is because of the increase in LBPA? or am I getting that wrong?

Hyunjung Jade Lim: You are right. We think that extended dormancy produces numerous vesicles that need to be removed via the formation of multivesicular bodies. Increased formation of multivesicular bodies was assessed by LBPA accumulation here. 
Unknown questioner: I wanted to ask a naïve question. Is there any possibility that the multivesicular bodies are doing something to actually help support the embryo while in diapause? Is it getting some nutrients or some benefit from this?

Hyunjung Jade Lim: They may use this system to their benefit initially, but if it goes on for too long, they may end up dying or getting sick, as in many other systems. Too much autophagy has a negative effect on cell survival.

Bruce Murphy: Your multivesicular bodies, could they also be delivery, is that only cell waste or is there delivery by some extracellular vesicles?

Hyunjung Jade Lim: Multivesicular bodies are actually associated with both waste removal and vesicle trafficiking. So they could also be delivering something to other cells.

Bruce Murphy: So they could serve in a two-way communication between the embryo and the uterus?

Hyunjung Jade Lim: Yes, I think that's possible.

Bruce Murphy: Any inhibitor of FGF How many FGFs are there? 18 or 20?

Hyunjung Jade Lim: There are many different FGFs, but there are four receptors for them. Blastocysts express these receptors and that's why we chose this FGF antagonist.

Unknown questioner: So my question is a little bit related. I maybe was confusing things, but I actually wanted to ask how your study can be correlated to the exosomes that we talked about yesterday, because the question I wanted to ask yesterday to the speaker was actually whether the exosomes could be rather disposed of waste products instead of communication.

Hyunjung Jade Lim: Exosomes are associated with both waste disposal and cell-cell communications. In order to answer the question as to what roles these exosome play during embry-uterine interactions, one has to isolate exosomes from pure cell source and identify what's in there. This is something we are interested to do, but it would be every challenging to do because only a minute amount of material is available from embryos. 